\title{
Local Keypoint-based Faster R-CNN
}

Xintao Ding ${ }^{1,2, *}$ Qingde $\mathrm{Li}^{3,{ }^{*}}$ Yongqiang Cheng ${ }^{3}$ Jinbao Wang $^{1,2}$ Weixin Bian ${ }^{1,2}$ Biao Jie $^{1,2}$

${ }^{1}$ School of Computer and Information, Anhui Normal University, Wuhu, China

${ }^{2}$ Anhui Province Key Laboratory of Network and Information Security, Wuhu, China

${ }^{3}$ School of Engineering and Computer Science, University of Hull, Hull, HU67RX, UK

${ }^{*}$ Corresponding author

Abstract. Region-based Convolutional Neural Network (R-CNN) detectors have achieved state-ofthe-art results on various challenging benchmarks. Although R-CNN has achieved high detection performance, the research of local information in producing candidates is insufficient. In this paper, we design a Keypoint-based Faster R-CNN (K-Faster) method for object detection. K-Faster incorporates local keypoints in Faster R-CNN to improve the detection performance. In detail, a sparse descriptor, which first detects the points of interest in a given image and then samples a local patch and describes its invariant features, is first employed to produce keypoints. All 2-combinations of the produced keypoints are second selected to generate keypoint anchors, which are helpful for object detection. The heterogeneously distributed anchors are then encoded in feature maps based on their areas and center coordinates. Finally, the keypoint anchors are coupled with the anchors produced by Faster R-CNN, and the coupled anchors are used for Region Proposal Network (RPN) training. Comparison experiments are implemented on PASCAL VOC 07/12 and MS COCO. The experimental results show that our K-Faster approach not only increases the mean Average Precision (mAP) performance but also improves the positioning precision of the detected boxes.

Keywords - Keypoint; SIFT; Convolutional Neural Network; Faster R-CNN

\section{Introduction}

General object detection is a complex problem. One of the main tasks for object detection is the localization problem, which is used to assign accurate bounding boxes to different objects [1]. In the last two decades, object detectors based on Convolutional Neural Networks (CNNs) [2-5] have achieved state-of-the-art results on various challenging benchmarks [6-8]. As two representative Region-based CNN (R-CNN) methods, both Fast/Faster R-CNN [3, 4] and Region-based Fully Convolutional Network (R-FCN) [9] use a Region Proposal Network (RPN) to generate region proposals. RPN initializes anchors of different scales and aspect ratios at each convolutional feature map location [4]. Although the anchor potentially covers the object of interest, it does not focus on local information. When a human identifies an object, both global structural information and local individual information are used in the identification [10].

Our work is motivated by the following two questions. First, is it possible to use local information to generate region proposals? Second, if the first answer is positive, are the generated proposals helpful for the performance of object detection? To meet this goal, we employ a keypoint as a kind of local information and fuse it in RPN in this study.

Fig. 1 shows an example of object detection by combining Faster and keypoint anchors. Faster RCNN cannot produce proposals for the bus, partially due to the error between the ground truth and the initialized anchor box. Although Faster R-CNN uses bounding-box regression to adjust the error from 
an anchor box to a ground-truth box, the regression may be powerless when the error between them is too large. By combining the two kinds of anchors, both the bus and car in Fig. 1a can be correctly detected.

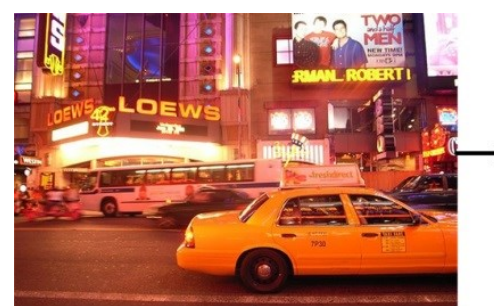

(a) Input image

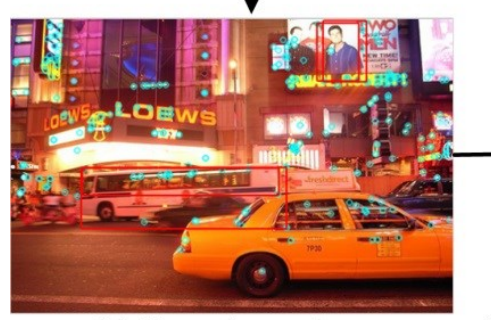

(c) Keypoint anchors

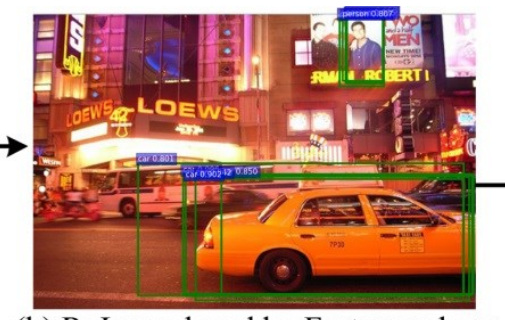

(b) RoIs produced by Faster anchor

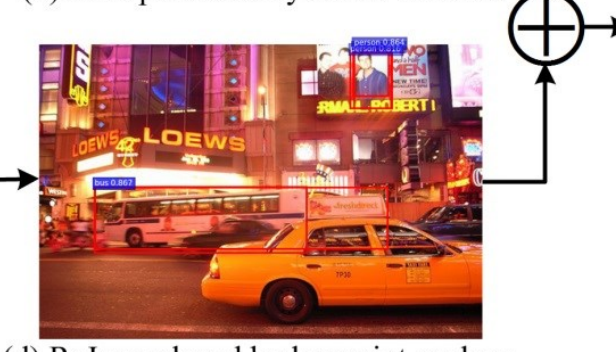

(d) RoIs produced by keypoint anchors

Fig. 1 An example of object detection by combining Faster and keypoint anchors.

In this work, we propose the keypoint-based Faster R-CNN method (K-Faster), which incorporates local keypoints in Faster R-CNN for object detection. All 2-combinations of the produced keypoints on an image are selected to generate bounding boxes, which are keypoint anchors (Fig. 1c). Every keypoint anchor is arranged in a convolutional network based on an Area Ratio of the Anchor to the Image (ARoAI) and the center coordinates of the anchor. On the one hand, a feature map with a lower index is designed to encode an anchor with a greater ARoAI, while a feature map with a higher index is used to encode an anchor with a smaller ARoAI. On the other hand, a grid point in a feature map is used to encode an anchor if the center coordinates of the anchor are convolved to the grid. The keypoint anchors together with Faster anchors, which are boxes with fixed scales and aspect ratios [4], are trained by RPN to produce proposals. Our approach employs the keypoint as a kind of local information and fuses it with Faster R-CNN for region proposal. The keypoints around the object may produce a keypoint anchor with a certain aspect ratio or size that cannot be covered by the anchors of Faster R-CNN. The keypoint anchor is helpful for region proposals, and the coupling anchors make the model have a more powerful ability. Extensive experiments implemented on PASCAL VOC 07/12 and MS COCO demonstrate that K-Faster can improve the detection performance.

Our main contributions are summarized as follows:

1. We incorporate local keypoints in Faster R-CNN to improve object detection. The designed keypoint anchors are coupled with Faster anchors to cover the deficiency of Faster R-CNN, which initializes anchor boxes with preset aspect ratios and sizes.

2. We design an area-based technology to encode anchors with a heterogeneous distribution. Because the keypoints are attracted to pixels with heterogeneous intensity, the heterogeneously distributed anchors are partitioned in groups using ARoAI and are encoded in feature maps for RPN training.

3. Compared with Faster R-CNN, our K-Faster approach not only increases the mean Average Precision (mAP) performance but also improves the positioning precision of the detected boxes. 


\section{Related work}

In recent years, object detection has achieved superior performance with the implementation of CNN [2-5, 11-13]. Generally, CNN-based object detection methods may be divided into two groups: (1) region proposal-based two-stage method and (2) the proposal-free one-stage method [14-16].

\subsection{Two-stage method}

R-CNN [17], Fast R-CNN [3], Faster R-CNN [4], R-FCN [9] and Mask R-CNN [12] are representative two-stage methods. Although R-CNN can be considered as a milestone of the twostage method for object detection, it classifies every region proposal separately and is time-consuming $[14,16]$. Fast R-CNN shares computation between the proposal extraction and classification steps using ROI-Pooling and therefore improves the efficiency greatly. Faster R-CNN designs RPN to generate proposals from anchor boxes and achieves further increases in speed and precision. R-FCN further improves speed and accuracy by removing fully connected layers and adopting positionsensitive score maps for final detection $[9,16]$. Mask R-CNN, which may be used for object instance segmentation and bounding box detection, extends Faster R-CNN by adding a branch that outputs the object mask [12].

Recently, many technologies have been designed to improve object detection. In order to combine multiple convolutional features, the multi-scale technology is employed for improvement $[18,19]$. Sean et al. designed an Inside-Outside Net (ION) [18], which concatenated multiple convolutional features to improve object detection. In [19], a Multi-scale Location-aware Kernel Representation (MLKP) is proposed to capture high-order statistics of deep features in proposals. Li et al. proposed a Zoom-out-and-In network for object Proposals (ZIP) and employed a Map Attention Decision (MAD) unit to search for neuron activations [20].

Since knowledge may be helpful for object detection, some studies focus on knowledge $[10][21][22]$. As shown in [10], the authors designed CoupleNet to couple the global structure with local parts for object detection. As a class of corner-based detector, DeNet evaluates the distribution of the box corners to refine bounding boxes for improvement [21]. Jiang et al. introduced an object co-detection method (CRFNet) that exploited contextual information among multiple images through a higher-order conditional random field [22].

\subsection{One-stage method}

Alternatively, one-stage detectors, such as You Only Look Once (YOLO) [5], Single Shot MultiBox Detector (SSD)[23], and CornerNet [24] detect objects in a single network. They are usually more computationally efficient than two-stage detectors [1][24]. Compared with two-stage methods, YOLO does not require proposals. YOLO forwards the input image once through a convolutional network to directly predict object classes and locations, and therefore it is extremely fast [5]. Combining dense anchor boxes and pyramid features, SSD directly classifies anchor boxes [23]. CornerNet, which uses an hourglass network as its backbone, detects each object bounding box as a pair of keypoints [24].

Many one-stage detectors share similarity with SSD. Similar to the frame work of SSD, the network structure of Deeply Supervised Object Detector (DSOD) is divided into two parts: the backbone sub-network for feature extraction and the front-end sub-network for prediction over multiscale response maps [16]. RetinaNet uses features pyramids occurred in SSD to address the extreme foreground-background class imbalance during training of dense detectors [25]. By attaching the 
Context Enhancement Blocks (CEBs) to the shallow layers in SSD, an advanced one-stage detector CEBNet is proposed in [26].

In this study, we focus on the improvement of Faster R-CNN using local keypoints. Although DeNet [21] and CornerNet [24] are point-based CNN methods, their flowcharts are complex. As a two-stage method, DeNet needs to jointly optimize the costs of both stages, i.e., the corner probability distribution, final classification distribution and bounding box regression cost [21]. The network of CornerNet may be divided in three modules: an hourglass backbone network and two prediction streams . In our design, the network modules are similar with Faster R-CNN. The main task of KFaster is to couple keypoint anchors with Faster anchors to cover the deficiency of Faster R-CNN, which initializes anchor boxes with preset aspect ratios and sizes.

\section{Proposed method}

\subsection{Overview of methodology}

The main design of our proposed method is to use local information to train candidates. In this study, we employ keypoint anchors to achieve the design. Fig. 2 shows the main design of our method. Fig. 2a shows the input image $I$ with a size $W \times H$. Fig. 2b shows the produced keypoints. Fig. 2c shows all the resulting anchor boxes, which total $C_{N}^{2}$, and $N$ is the number of total keypoints. To train an RPN, a positive or negative anchor with a high or low Intersection over Union (IoU) overlap with a ground-truth box, called the Anchor of Interest (AoI), should be assigned a positive or negative label. The bias between the anchor box and ground truth box, which is called the ground bias, should be calculated to supervise bounding box regression. To arrange the labels and ground biases to 3Dimensional (3-D) tensors, which serve as supervision information for the RPN, the anchors in Fig. $2 \mathrm{c}$ are partitioned into $k_{S}$ groups based on the $A R o A I$, as shown in Fig. $2 \mathrm{~d}$. All labels of the AoIs in each group are arranged on a corresponding 2-Dimensional (2-D) feature map, which indicates the convolution results of one channel [27] (Fig. 2e). For every AoI, its label is mapped to a layer position based on the coordinates of the anchor center (Fig. 2e). Every ground bias is parameterized by 4 coordinates and therefore is encoded on 4 feature maps in a similar way, as shown in Fig. 2 f. Figs. $2 g$ and $2 \mathrm{~h}$ are label and ground bias tensors obtained from Faster R-CNN, respectively. Fig. 2i shows supervision information for RPN, and Figs. $2 \mathrm{i} 1$ and $2 \mathrm{i} 2$ show coupled tensors. Based on the integrated supervision information, our method follows a network similar to that of Faster R-CNN. 


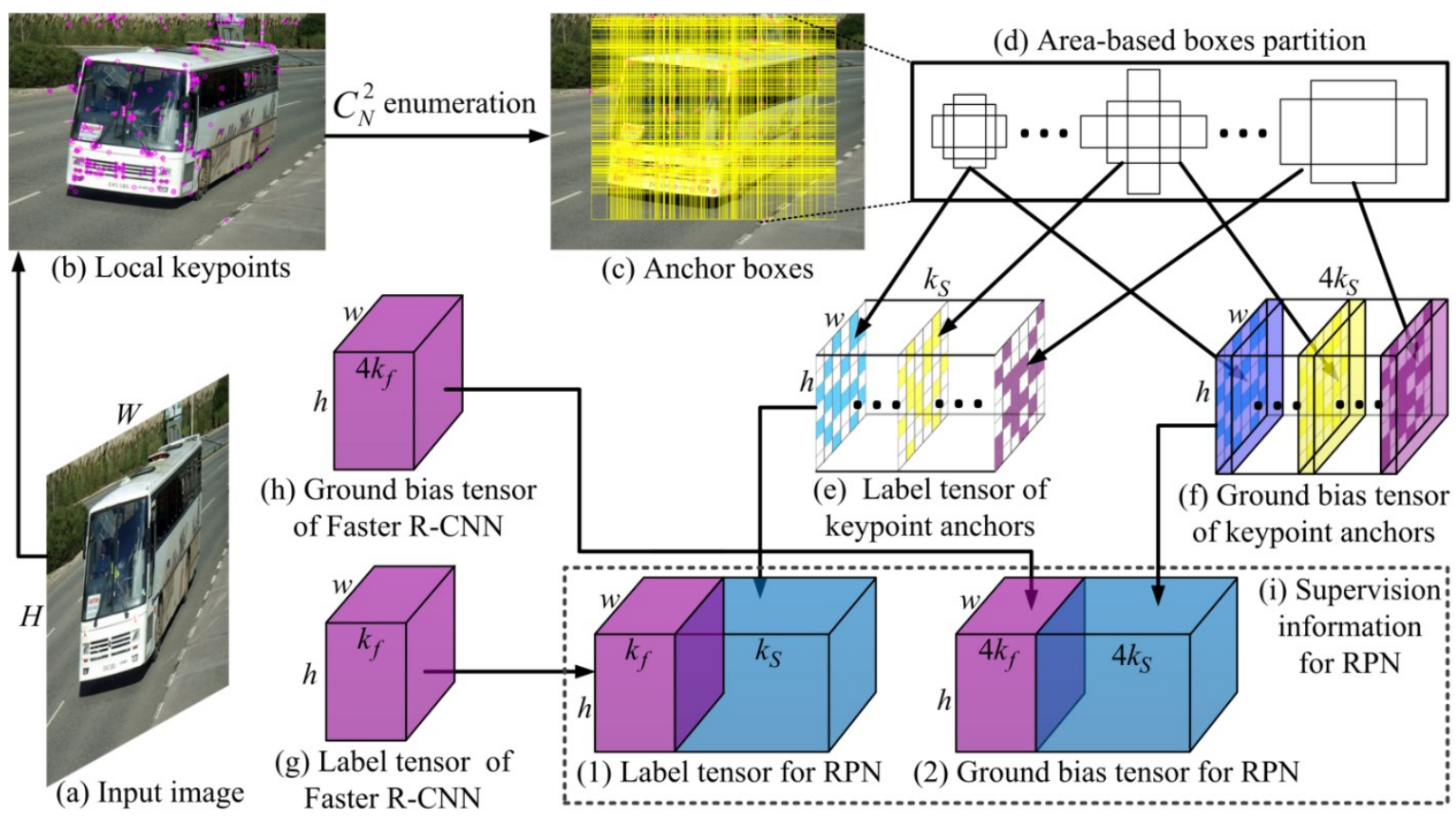

Fig. 2. Flowchart of the construction of the supervision information for RPN. (a) Input image. (b) Local keypoints. (c) The resulting anchor boxes after enumerating all 2-combinations. (d) Area-based boxes partition. (e) 3-D label tensor induced by the keypoints. (f) 3-D ground bias tensor induced by keypoints. (g) and (h) are label and bias tensors of Faster R-CNN, respectively. (i) The supervision information of our method for RPN. (i1) and (i2) show label and bias tensors for RPN, respectively.

\subsection{Keypoint detection}

In order to improve the smartness of the region proposal, the keypoint, which involves local information, is employed to improve the coverage ability of anchors. Generally, the keypoint is coupled with a descriptor. In this work, a sparse descriptor, which first detects the points of interest in a given image and then samples a local patch and describes its invariant features [28], is employed to produce the keypoint.

During the last two decades, a large variety of sparse descriptors have been developed, including classical Scale-Invariant Feature Transform (SIFT) [29] and Speeded-Up Robust Feature (SURF) [30] descriptors, binary descriptors [31], and learning-based descriptors [32][33]. Although learning methods can achieve great accuracy, they usually produce large dimension features and are computationally expensive [32][33]. In addition, the preparation of substantial data for training is not a trivial subject. In order to produce as many AoI as possible, a robust keypoint detector that detects as many corresponding keypoints on the image is needed. Although binary descriptors are usually proposed in a simple presentation, they may be insufficient for producing massive keypoints based on experiments in [34]. Although SURF is more efficient for registration types of tasks, it is insufficient for producing enough keypoints [34]. In this paper, a Global OPtimized SIFT (GOP), which was demonstrated to produce many more keypoints in [34], is employed for keypoint detection.

\subsection{Generating keypoint anchors}

All 2-combinations of the produced keypoints are enumerated to generate anchors. Let the number of keypoints produced by GOP be $N_{G}$. Because multiple descriptors may be produced at one 
keypoint [29], the same keypoints are merged into one keypoint. Let the keypoints after merging be $P_{K}=\left\{p_{i}^{0}=\left(x_{i}^{0}, y_{i}^{0}\right) \mid i=0,1, \cdots\right.$

$\square^{\prime}$, For a 2-combination of points $\left(p_{i}^{0}, p_{j}^{0}\right)$ extracted from $P_{K}$, let its corresponding anchor be $a_{i j}^{0}=\left(x_{i}^{0}, y_{i}^{0}, x_{j}^{0}, y_{j}^{0}\right)$, where $x_{i}^{0} \leq x_{j}^{0}, y_{i}^{0} \leq y_{j}^{0}$, $\left(x_{i}^{0}, y_{i}^{0}\right),\left(x_{j}^{0}, y_{j}^{0}\right) \in P_{K}$. The anchor $a_{i j}^{0}$ with small area should be ignored because a proposal with small area is filtered in the stage of proposal refinement [4]. Let $A=A_{\text {fas }} \cup A_{\text {des }}$ be the final resulting anchors, where $A_{\text {fas }}$ is the set of anchors produced by Faster R-CNN; $\left.\left.A_{d e s}=\left\{a_{i j}^{0} \mid s\left(a_{i j}^{0}\right) \geq T_{s a}\right\} \square^{\prime}, \quad y_{i}, x_{j}, y_{j}\right)\right\}$ is the set of anchors produced by keypoints, $s(\bullet)$ is the pixel area of $\bullet$, and $T_{s a}$ is a threshold.

\subsection{Encoding keypoint anchors}

To generate region proposals, a box-regression layer (reg) and a box-classification layer ( $c l s)$ are used to map the coordinates and scores of the proposals in the framework of Faster R-CNN [4]. In this study, we integrate keypoint and Faster R-CNN anchors to design reg and cls. The label and ground bias induced by the keypoint should be mapped in 3-D tensors as the supervision information to train reg and $\mathrm{cls}$.

Let $f m_{R P N}=\{(r, c) \mid r=0,1, \cdots$ be the 2-D feature map of the last shared convolutional layer; $h$ and $w$ are respectively the numbers of rows and columns of the map. Let the labels fed to cls be a 3-D tensor, denoted as $L t=\left\{(l, r, c) \mid l=0,1, \cdots \quad \ldots \quad \ldots \quad \ldots \quad\right.$, where $k_{c}=k_{f}+k_{S}, k_{S}$ and $k_{f}$ are respectively the number of maximum possible proposals induced by keypoints and Faster R-CNN at each point of $f m_{R P N}$. Lt may be decomposed into two sub-tensors, as shown in (1):

$$
L t=L t_{f a s} \cup L t_{d e s}
$$

where $L t_{\text {fas }}=L t\left(l<k_{f}\right)$ is the part of the tensor induced by Faster R-CNN (Fig. 2g) and $L t_{d e s}=L t\left(k_{f} \leq l<k_{c}\right)$ is the part of the tensor induced by the keypoint (Fig. 2e). Furthermore, the 3-D $L t_{d e s}$ may be expressed as a set of 2-D feature maps, as shown in (2).

$$
L t_{d e s}=\bigcup_{t=0}^{k_{S}-1}
$$

where $f m_{d e s}^{t}$ is the feature map of the $t$-th channel. 
In order to arrange the label and ground bias in 3-D tensors, we partition the set of keypoint anchors $A_{d e s}$ into $k_{S}$ groups, as shown in (3):

$$
A_{\text {des }}=\bigcup_{t=0}^{k_{S}-1}
$$

where if $a_{i j} \in A_{d e s}^{t}$, then

$\operatorname{ARoAI}\left(a_{i j}\right)=\frac{s_{T}}{s\left(a_{i j}\right)} \in\left\{\begin{array}{l}\left(2^{t}, 2^{t+1}\right], t=0,1, \cdots \\ \left(2^{k_{S}-1},+\infty\right), t=k_{S}-1\end{array}\right.$

$S_{T}$ is a threshold that is the base size of the image set.

Combining (2) and (3), we map $a_{i j}=\left(x_{i}, y_{i}, x_{j}, y_{j}\right) \in A_{d e s}^{t}$ to the $t$-th feature map $f m_{d e s}^{t}$ at position $p_{i j}^{c}=\left(\frac{x_{i}+x_{j}}{2 r}, \frac{y_{i}+y_{j}}{2 r}\right) \sqcup \quad, \quad$, , where $r=[H / h]$ is the feature stride from the input image to the feature map $f m_{R P N}$.

The ground bias tensor $B t=\left\{\left(l_{b}, r, c\right) \mid l_{b}=0,1, \cdots \quad\right\}$ may be decomposed into two bias tensors, as shown in (4).

$$
B t=B t_{f a s} \cup B t_{d e s},
$$

where $B t_{f a s}=B t\left(l_{b}<4 k_{f}\right)$ is the part of tensor induced by Faster R-CNN (Fig. 2h) and $B t_{d e s}=B t\left(4 k_{f} \leq l_{b}<4 k_{c}\right)$ is the part of the tensor induced by the keypoint (Fig. 2f). $B t_{d e s}$ may be expressed as (5):

$$
B t_{d e s}=\bigcup_{t=0}^{4 k_{s}-1}
$$

Combining (3) and (5), for any $a_{i j} \in A_{d e s}^{t}$, we map its ground biases to $f m_{d e s}^{4 t}, f m_{d e s}^{4 t+1}, f m_{d e s}^{4 t+2}$, and $f m_{d e s}^{4 t+3}$ at the position $p_{i j}^{c}$.

For $a_{i j} \in A_{f a s}$, the mappings of $L t_{f a s}$ and $B t_{f a s}$ are similar to those in [4]. After the label and ground biases of all $a_{i j}$ are mapped in their corresponding tensors, the 3D tensors $L t$ and $B t$ serve as supervision information to train the RPN. 


\subsection{The encoding steps}

In this Section, we present encoding steps of our proposed method. Table 1 shows the input variables and comments used in this Section. $P_{K}$ and $k_{S}$ are two key parameters of our method. $k_{f}$ is the parameter for anchors of Faster R-CNN. $b_{g t}, w$, and $h$ are parameters produced by input image. The other four variables are thresholds for RPN training.

Table 1 Variables used in our method.

\begin{tabular}{ll}
\hline Variable & Comment \\
\hline$P_{K}$ & A set with $N_{K}$ keypoints \\
$k_{S}$ & The number of channels of the label tensor induced by keypoints \\
$k_{f}$ & The number of channels of the label tensor induced by Faster R-CNN \\
$b_{g t}$ & The array of ground truth boxes with the size of $4 \times N_{g t}$ \\
$w$ & The number of columns of the last shared convolutional tensor \\
$h$ & The number of rows of the last shared convolutional tensor \\
$T_{R P N_{-} p o}$ & The IoU threshold for positive anchors \\
$T_{R P N_{-} n o}$ & The IoU threshold for negative anchors \\
$T_{R P N_{-} b s}$ & The threshold of the number of anchors for RPN training \\
$r_{R P N_{-} f g}$ & The ratio of the anchors that are labeled as foreground \\
\hline
\end{tabular}

For convenience, Fig. 3 shows the illustration of our mapping. Let the Faster anchors of be an array $A_{\text {fas }} \in \square^{\prime \prime}$ " , the keypoint anchors be an array $A_{\text {des }} \in \square$ " ' Then

$$
A=\left[\begin{array}{c}
A_{\text {fas }} \\
A_{d e s}
\end{array}\right]_{\left(N_{a f}+N_{a d}\right) \times 4}
$$

is the array of all the anchors, which is coupled vertically by $A_{\text {fas }}$ and $A_{\text {des }}$ (Fig. 3a). Let

$$
A^{\prime}=\left[\begin{array}{c}
A_{f a s}^{\prime} \\
A_{d e s}
\end{array}\right]_{\left(n_{a f}+N_{a d}\right) \times 4},
$$

which is resulted from $A$ after removing the anchors that are not inside the image (Fig. 3b). Let a query list between $A$ and $A^{\prime}$ be $q A$, where the $k$-th anchor in $A^{\prime}$ corresponds to the $q A_{k}$-th anchor in $A$ (Figs. 3a and 3b). The labels of $A^{\prime}$ are initialized to $l A^{\prime}=\left[l A_{\text {fas }}^{\prime} l A_{d e s}\right]=(-1, \cdots \quad \square \quad$, where $l A_{\text {fas }}^{\prime} \in \square \quad l A_{\text {des }} \in \square^{\cdots}$.

Let $L t_{\text {fas }}^{1} \in \square^{\cdots .2}$ and $L t_{d e s}^{1} \in \square^{\prime \prime}$ be 1-Dimensional (1-D) label tensors, which are respectively reshaped from 3-D tensors $L t_{f a s}$ and $L t_{d e s}$ in the dimension order of channel, width, and height, as shown the red arrowed line in Fig. 3d. Then the 1-D label tensor of $L t$ is 
$L t^{1}=\left[L t_{\text {fas }}^{1} L t_{\text {des }}^{1}\right] \in \square^{\prime \prime}$, , which is initialized to $L t^{1}=(-1, \cdots \quad$. In order to map the anchors in the list of $A_{d e s}$ to the 1-D tensor $L t_{d e s}^{1}$ introduced in Section 3.4, a query list

$$
l q\left(\operatorname{ind}\left(a_{i, j}\right)\right)=\operatorname{row}_{i j}+\operatorname{col}_{i j}+c h a_{i j}
$$

is designed to map the ind $\left(a_{i, j}\right)$-th anchor in $A_{d e s}$ to the $l q\left(\operatorname{ind}\left(a_{i, j}\right)\right)$-th label in the 1-D tensor $L t_{d e s}^{1}$, where $\operatorname{ind}\left(a_{i, j}\right)$ is the index of $a_{i, j}$ in $A_{d e s} ; \quad \operatorname{row}_{i j}=w k_{S} y_{i j}^{c} ; \quad \operatorname{col}_{i j}=k_{S} x_{i j}^{c}$; $\operatorname{ch} a_{i j}=\underset{t}{\arg }\left[\operatorname{ARoAI}\left(a_{i j}\right) \in\left(2^{t}, 2^{t+1}\right]\right]$.

Figs. 3c-3e illustrate the mapping from the list of keypoint anchors to the label tensors. Take the 0th keypoint anchor $a_{01}$ (Fig. 3c) for example, its label is arranged in the 3-D tensor $L t_{d e s}$ at $\left(x_{01}^{c}, y_{01}^{c}, t\right)$, as shown the yellow square in Fig. $3 \mathrm{~d}$, where $\left(x_{01}^{c}, y_{01}^{c}\right)=p_{01}^{c}$ corresponds to the center coordinate of $a_{01}, t$ is obtained by $A R O A I$. After fetching the elements along with the red arrowed line in Fig. 3d, the 3-D tensor $L t_{d e s}$ (Fig. 3d) is reshaped to 1-D tensor $L t_{d e s}^{1}$ (Fig. 3e). The label of the 0th keypoint anchor (the blue item in Fig. 3c) is arranged to $L t_{d e s}^{1}$ at $l q(0)=w k_{S} y_{01}^{c}+k_{S} x_{01}^{c}+t$ (the yellow item in Fig. 3e).

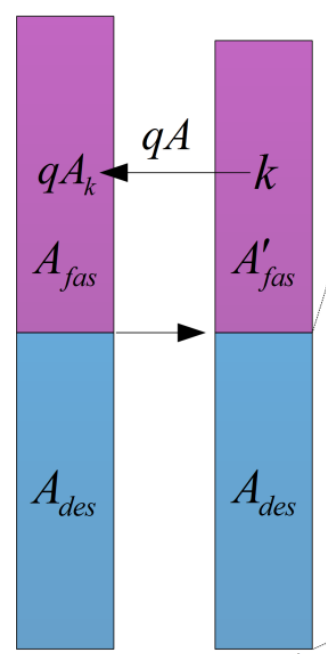

(a) $A$

(b) $A^{\prime}$

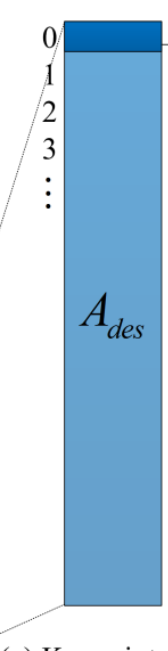

(c) Keypoint anchors

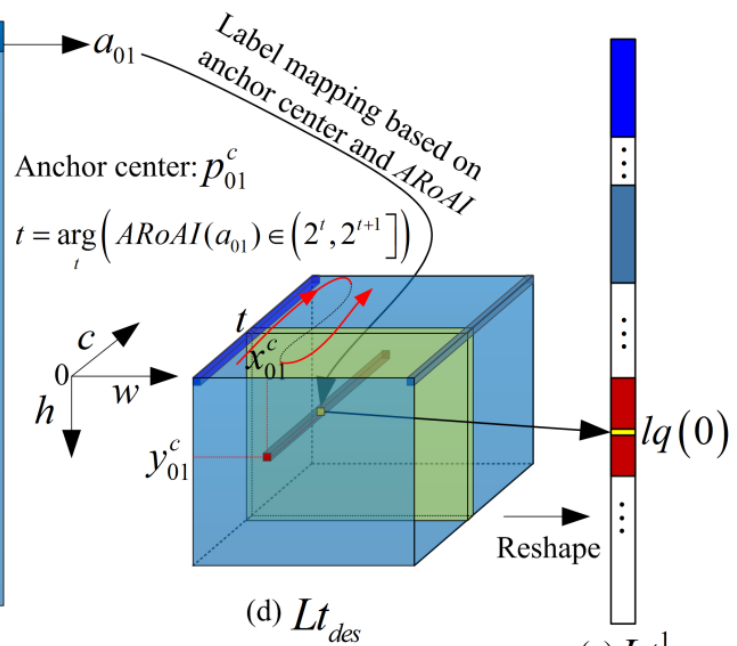

(e) $L t_{d e s}^{1}$

Fig. 3. Illustration of the mapping from anchor to tensor. (a) Array of all the anchors $A$. (b) Array of all the anchors inside image $A^{\prime}$. (c) The list of keypoint anchors. (d) 3-D label tensor $L t_{d e s}$. (e) 1-D label tensor $L t_{d e s}^{1}$.

Let the overlapping array generated by the anchors and the ground truth boxes be $O=\left(o_{i j}\right)_{\left(n_{a f}+N_{a d}\right) \times N_{g t}}$, where $o_{i j}$ is the overlap between the $i$-th anchor of $A^{\prime}$ and the $j$-th ground truth box in $b_{g t}$. Let the row maximums of $O$ be a vector $r_{\max } \in \square^{{ }^{\prime} r}$, i.,e., the $k$-th element $r_{k}$ of $r_{\max }$ is 
the maximum of the $k$-th row in $O$; Let the anchor indexes of the column maximums of $O$ be the vector $c_{\max } \in \square^{\wedge}$, i.e., the $k$-th element $c_{k}$ of $c_{\max }$ is the index of the maximum of the $k$-th column in $O$. Then, the indexes of the anchors with positive label in $A^{\prime}$ for RPN training can be obtained as follows:

(1.1) Let $l A_{i}^{\prime}=1$ if $r_{i} \geq T_{R P N_{-} p o}$ or $i=c_{k}$. Obtain all the indexes of the anchors with the positive label 1, i.e., ind $\left(l A^{\prime}=1\right)=\left(i_{1}, i_{2}, \cdots\right.$;

(1.2) Let $T_{R P N_{-} f g}=\left[r_{R P N_{-} f g} T_{R P N_{-} b s}\right]$. Randomly choose $T_{R P N_{-} f g}$ elements in ind $\left(l A^{\prime}=1\right)$ if the number of the assigned positive label in (1.1) is greater than $T_{R P N_{-} f g}$. Let $p a_{T_{R P N_{-} f g}} \subset$ ind $\left(l A^{\prime}=1\right)$ be the set of selection;

(1.3) The index vector of the anchors with positive label in $A^{\prime}$ for RPN training is $\left.p a \square \quad, \ldots x_{d e s}\right]$, where $p a=p a_{T_{R N_{-} f g}}$ if $(1.2)$ is executed, otherwise $p a=\operatorname{ind}\left(l A^{\prime}=1\right)$.

The indexes of the anchors with negative label in $A^{\prime}$ for RPN training are obtained as follows:

(2.1) Let $l A_{i}^{\prime}=0$ if $r_{i}<T_{R P N_{-} n o}$. Obtain all the indexes of the anchors with the negative label 0, i.e., $\operatorname{ind}\left(l A^{\prime}=0\right)=\left(i_{1}, i_{2}, \cdots\right.$;

(2.2) Let $T_{R P N_{-} b g}=T_{R P N_{-} b s}-n p$. Randomly choose $T_{R P N_{-} b g}$ elements in ind $\left(l A^{\prime}=0\right)$ if the number of the assigned negative label is greater than $T_{R P N_{-} b g}$. Let $n a_{T_{R P N_{-} b g}} \subset$ ind $\left(l A^{\prime}=0\right)$ be the set of selection;

(2.3) The index vector of the anchors with negative label in $A^{\prime}$ for RPN training is $n a=n a_{T_{R P N} b g}$ if (2.2) is executed, otherwise $n a=\operatorname{ind}\left(l A^{\prime}=0\right)$.

Based on $p a_{\text {fas }}(i)$ and $n a_{\text {fas }}(i)$, i.e., the positive and negative indexes of $A_{\text {fas }}^{\prime}$ in Fig. 3b, the 1-D label tensor $L t_{\text {fas }}^{1}$ is obtained using query list $q A$ :

(3.1) For every $p a_{f a s}(i)$, let the $q A\left(p a_{\text {fas }}(i)\right)$-th label in $L t_{\text {fas }}^{1}$ be 1, i.e., $L t_{\text {fas }}^{1}\left(q A\left(p a_{\text {fas }}(i)\right)\right)=1$;

(3.2) For every $n a_{\text {fas }}(i), L t_{\text {fas }}^{1}\left(q A\left(n a_{\text {fas }}(i)\right)\right)=0$.

Based on $p a_{d e s}(i)$ and $n a_{d e s}(i)$, i.e., the positive and negative indexes of $A_{d e s}$ in $A^{\prime}$ (Fig. 3b), the 1-D label tensor $L t_{d e s}^{1}$ is obtained using query list $l q$ : 
(4.1) For every $p a_{d e s}(i)$, the label of the $p a_{d e s}(i)-n_{a f}$-th anchor in $A_{d e s}$ is 1 . Therefore, let the $l q\left(p a_{d e s}(i)-n_{a f}\right)$-th label in $L t_{d e s}^{1}$ be 1, i.e., $L t_{d e s}^{1}\left(l q\left(p a_{d e s}(i)-n_{a f}\right)\right)=1$;

(4.2) For every $n a_{d e s}(i), L t_{d e s}^{1}\left(l q\left(n a_{d e s}(i)-n_{a f}\right)\right)=0$.

For the 2-D array of ground bias $B t^{2} \in \square^{\ldots . t h \cdots 1 \ldots 1}$, it is initialized to $B t^{2}=0$. In order to evaluate the loss of the bounding box regression, the regressions of the anchors with positive and negative labels are weighted. Let $W_{i n}^{2}=W_{o u t}^{2}=0 \in \square^{\cdots+1}$ be the initialization of the positive and negative weights. All the four regressions of the $i$-th row in $W_{\text {in }}^{2}$ are assigned with weight 1 if the $i$-th label of $L t^{1}$ is 1 . And the rows of $W_{\text {out }}^{2}$ corresponding to negative anchors with the label 0 are assigned with weight $T_{p o s}=1 / \operatorname{Card}\left(L t^{1} \geq 0\right), \operatorname{Card}(\bullet)$ is the cardinality of $\bullet$.

The encoding steps of the supervision information for RPN training are as follows. $L t, B t, W_{\text {in }}$, and $W_{\text {out }}$ are outputs, where $L t$ and $B t$ are label and ground bias tensors, respectively; $W_{\text {in }}$ and $W_{\text {out }}$ are weight tensors used to evaluate the loss of the bounding box regression.

Step 1: Generate the array of Faster anchors $A_{\text {fas }}$.

Step 2: Generate the keypoint anchors $A_{\text {des }}$ based on Section 3.3.

Step 3: Result in $A$ and $A^{\prime}$ based on (6) and (7), respectively.

Step 4: Build a query list $q A$ between $A$ and $A^{\prime}$.

Step 5: Build a query list $l q\left(\operatorname{ind}\left(a_{i, j}\right)\right)$ from the anchors in $A_{d e s}$ to the labels in the 1-D tensor $L t_{d e s}^{1}$ based on (8).

Step 6: Assign the overlapping array $O$, and result in $r_{\max }$ and $c_{\max }$.

Step 7: Obtain the indexes of the anchors with positive and negative labels in $A^{\prime}$ for RPN training based on (1.1)-(1.3) and (2.1)-(2.3), respectively.

Step 8: Assign anchor labels to $L t_{\text {fas }}^{1}$ and $L t_{\text {des }}^{1}$ based on (3.1)-(3.2) and (4.1)-(4.2), respectively.

Step 9: Reshape $L t_{\text {fas }}^{1}$ and $L t_{\text {des }}^{1}$ to 3-D tensors $L t_{\text {fas }}$ and $L t_{\text {des }}$ respectively in the dimension order of channel, width, and height. Couple the two tensors to $L t$, as shown in (1).

Step 10: Compute the ground bias of all the anchors in $A^{\prime}$, and assign them to $B t^{2}$ using query lists $q A$ and $l q$. 
Step 11: Obtain $W_{\text {in }}^{2}$ and $W_{\text {out }}^{2}$.

Step 12: Separate $B t^{2}, W_{\text {in }}^{2}$, and $W_{\text {out }}^{2}$ vertically into two parts that are respectively in size of $w h k_{f} \times 4$ and $w h k_{S} \times 4$. Reshape and couple them to 3-D tensors $B t, W_{\text {in }}$, and $W_{\text {out }}$ similar to Step 9.

\section{Experiments}

We evaluate our method on three datasets: PASCAL VOC 2007 [8], PASCAL VOC 2012 [8], and MS COCO [6]. Our experiments are implemented based on the framework of Faster R-CNN [4]. Both VGG16 [11] and ResNet101 [13] are employed as our backbone networks. The VGG16-based and ResNet101-based experiments are carried out respectively on Caffe [35] and TensorFlow [36]. We train and test networks on images of a single scale in which the shorter side is $s=600$ pixels [4]. The publicly available VGG16 and ResNet101 models pre-trained on ImageNet [2, 7] are used for corresponding initialization. We use a 1-GPU implementation, and thus the mini-batch size of RPN is 1. The models are trained starting from conv3 1 using an end-to-end schedule. The momentums are set as 0.9. The weight decay of K-Faster based on VGG16 is set to 0.0005 and that of K-Faster based on ResNet101 is set to 0.0001 . The mAP is primarily used to evaluate the detection performance.

\subsection{Implementation detail}

We tune the parameters on PASCAL VOC. The models are trained on the union set of VOC 2007 trainval and VOC 2012 trainval ("07+12"). They are evaluated on VOC 2007 test set. We initialize a learning rate of 0.001 and make the learning rate drop 10 times after every 50k iterations on the $07+12$ dataset. A total of $140 \mathrm{k}$ training iterations are run.

In addition to thresholds $T_{s a}$ and $s_{T}$, two main parameters $N_{G}$ and $k_{S}$ are introduced in this work.

$N_{G}$ and $k_{S}$ are the number of keypoints and the number of the channels of the keypoint-based label tensor, respectively. In our design, $T_{s a}$ is set to 16 to filter out small keypoint anchors, and $s_{T}$ is set to $600 \times 1000$, which is the multiplication of the lower boundary and upper boundary of the input image size. For $k_{S}$, it is appropriate to let it be 16 when $s_{T}=600 \times 1000$ because the anchors with the size less than $16 \times 16$ are convoluted to 1 point in the last shared convolutional layer. In detail, the anchors with the size less than $600 \times 1000 / 2^{15} \approx 18.3$ are mapped to the $k_{S}-1=15$-th feature map. In addition, because the tensor induced by the keypoint anchors is coupled with the tensor induced by Faster anchors for RPN training, corresponding parameters of K-Faster may be different from those of Faster R-CNN. The corresponding parameters are the number of anchors chosen for RPN training in an image $\left(N_{B S}^{R P N}\right)$, the number of regions of interest in an image for classification $\left(N_{R o I}\right)$, the number of top scoring boxes to keep before applying Non-Maximum Suppression (NMS) to RPN proposals 
( $N_{N M S}^{\text {pre }}$ ), and the number of top scoring boxes to keep after applying NMS to RPN proposals $\left(N_{N M S}^{\text {post }}\right)$. Above all, $N_{G}, N_{B S}^{R P N}, N_{R o I}, N_{N M S}^{p r e}$, and $N_{N M S}^{\text {post }}$ are the five parameters that we tune in this work.

\subsubsection{The parameter $N_{G}$}

We run VGG16-based experiments to evaluate $N_{G}$ using the encoding steps introduced in Section 3.5. We first train seven different models on seven levels of $N_{G}$ with $N_{B S}^{R P N}=448, N_{R o I}=128$, $N_{N M S}^{\text {pre }}=18 \mathrm{k}$, and $N_{N M S}^{\text {post }}=2 \mathrm{k}$. Every model is then tested with seven groups of parameters $\left(N t_{N M S}^{\text {pre }}\right.$, $N t_{N M S}^{\text {post }}$ ), where $N t_{N M S}^{\text {pre }}$ and $N t_{N M S}^{\text {post }}$ are respectively the number of top-scored RPN proposals before and after applying NMS in the stage of detection. The seven groups of $\left(N t_{N M S}^{\text {pre }}, N t_{N M S}^{\text {post }}\right)$ are $(6 \mathrm{k}, 300),(9 \mathrm{k}$, $300),(9 \mathrm{k}, 450),(12 \mathrm{k}, 600),(15 \mathrm{k}, 750),(18 \mathrm{k}, 750)$, and $(18 \mathrm{k}, 900)$. Table 2 summarizes the key results on the seven models. The test results with the greatest mAP are listed in the line of mAP in Table 2. The lines of Rec, Pre, $N t_{N M S}^{\text {pre }}$, and $N t_{N M S}^{\text {post }}$ in Table 2 are respectively the recalls, precisions, and testing parameters that correspond to the greatest mAP. Together with ground truth, the true positive and false positive samples are used to calculate the recall and precision on every class. A predicted detection is regarded as a true positive if the predicted class label is the same as the ground truth label and the IoU between the predicted bounding box and the ground truth one is greater than 0.5, otherwise the detection is a false positive one. The results of Rec and Pre listed in Table 2 are the averages of the recalls and precisions over all the classes.

Table 2. Experiments of $N_{G}$ on the PASCAL VOC 2007 test set.

\begin{tabular}{llllllll}
\hline$N_{G}$ & 120 & 160 & 200 & 300 & 400 & 500 & 600 \\
\hline$m A P(\%)$ & 75.8 & 76.2 & 76.2 & 76.1 & 76.2 & 75.8 & 76.1 \\
$\operatorname{Rec}(\%)$ & 88.4 & 88.3 & 88.0 & 88.1 & 87.2 & 86.0 & 86.1 \\
$\operatorname{Pre}(\%)$ & 19.9 & 22.7 & 21.9 & 23.6 & 26.9 & 29.7 & 30.0 \\
$N t_{N M S}^{\text {pre }}$ & $18 \mathrm{k}$ & $18 \mathrm{k}$ & $12 \mathrm{k}$ & $15 \mathrm{k}$ & $15 \mathrm{k}$ & $15 \mathrm{k}$ & $18 \mathrm{k}$ \\
$N t_{N M S}^{\text {post }}$ & 900 & 900 & 600 & 750 & 750 & 750 & 900 \\
\hline
\end{tabular}

$N_{G}=400$ is chosen for our proposed method. The recall rate ranges roughly from $88.4 \%$ to $86.1 \%$. Simultaneously, the precision ranges from $19.9 \%$ to $30.0 \%$. It can be seen that a greater $N_{G}$ takes advantage in precision, but a greater $N_{G}$ results in a smaller recall rate. Although $N_{G}=160,200$, and 400 are all able to obtain a similar greatest mAP $76.2 \%, N_{G}=400$ brings a greatest precision (Table 2). Therefore, $N_{G}$ with a value of 400 is chosen for K-Faster.

\subsubsection{RPN parameters}

In this Section, we implement experiments on $07+12$ using VGG16-based network to tune $N_{B S}^{R P N}$, $N_{R o I}, N_{N M S}^{p r e}$, and $N_{N M S}^{\text {post }}$. For $N_{G}=400$, the number of the induced keypoint anchors ranges roughly 
from $40 \mathrm{k}$ to $60 \mathrm{k}$. The number of the keypoint anchors with $\mathrm{IoU} \geq 0.7$ ranges from zero to several hundreds. We tune the four parameters of K-Faster in the range of one to two times of those used in Faster R-CNN. For simplicity, the parameters $N t_{N M S}^{p r e}$ and $N t_{N M S}^{\text {post }}$ used for NMS in the stage of detection are set to $9 \mathrm{k}$ and 300 , respectively. Table 3 shows our main experimental results.

Table 3. Experiments of RPN parameters on the PASCAL VOC 2007 test set.

\begin{tabular}{lllllll}
\hline$N_{B S}^{R P N}$ & $N_{\text {RoI }}$ & $N_{N M S}^{\text {pre }}$ & $N_{N M S}^{\text {post }}$ & $\operatorname{Rec}(\%)$ & $\operatorname{Pre}(\%)$ & $\operatorname{mAP}(\%)$ \\
\hline 448 & 256 & $24 \mathrm{k}$ & $4 \mathrm{k}$ & 85.7 & 30.8 & 76.3 \\
512 & 256 & $24 \mathrm{k}$ & $4 \mathrm{k}$ & 85.3 & 30.7 & 75.3 \\
448 & 196 & $24 \mathrm{k}$ & $4 \mathrm{k}$ & 85.7 & 30.4 & 76.1 \\
448 & 256 & $18 \mathrm{k}$ & $4 \mathrm{k}$ & 85.4 & 33.7 & 75.7 \\
448 & 256 & $24 \mathrm{k}$ & $2 \mathrm{k}$ & 85.4 & 31.3 & 75.6 \\
\hline
\end{tabular}

As shown in the first and second lines in Table 3, the experimental levels of $N_{B S}^{R P N}$ are different but the other three parameters are the same. Both the recall and precision obtained by $N_{B S}^{R P N}=448$ are greater than those obtained by $N_{B S}^{R P N}=512$, therefore, $N_{B S}^{R P N}=448$ takes advantage in mAP. The onefactor-at-a-time experiments on $N_{R o I}$ are listed in the first and third lines in Table 3. It can be seen that $N_{R o I}=256$ is more appropriate than 196. Similarly, $N_{N M S}^{\text {pre }}=24 \mathrm{k}$ and $N_{N M S}^{\text {post }}=4 \mathrm{k}$ take advantage in mAP.

Extended experiments on the same four parameters based on ResNet101 show similar results. This is partially due to the same structure of their RPN. Overall, the RPN parameters $N_{B S}^{R P N}, N_{R o I}, N_{N M S}^{p r e}$, and $N_{N M S}^{\text {post }}$ are respectively tuned to be $448,256,24 \mathrm{k}$, and $4 \mathrm{k}$ for K-Faster.

\subsection{Results on PASCAL VOC}

In this Section, we demonstrate that local information is helpful in terms of object detection. We evaluate K-Faster on the PASCAL VOC 2007 detection benchmark [8] together with VOC 2012. For the experiments on the set of PASCAL VOC 2007 test, the $07+12$ dataset is used for training. For the PASCAL VOC 2012 test set, we use the 10k trainval+test images in VOC 2007 and 16k trainval images in VOC 2012 for training (“07++12”).

We initialize a learning rate of 0.001 , and make the learning rate drop 10 times after every $80 \mathrm{k}$ iterations on the $07+12$ dataset. The networks based on VGG16 and ResNet101 are respectively run 180k and 200k training iterations. Table 4 shows our experimental results on the test set of VOC 2007. The rows of K-Faster16 and K-Faster101 show the results of our method using VGG16 and ResNet101 as the backbone networks, respectively. The notation "-" shows that corresponding result is unavailable. 
Table 4. Results on the PASCAL VOC 2007 test set (\%). Training data: "07+12": VOC 2007 trainval together with VOC 2012 trainval.

\begin{tabular}{|c|c|c|c|c|c|c|c|c|c|c|c|c|c|c|c|c|c|}
\hline \multirow{2}{*}{$\frac{\text { Method }}{\text { One-stage detecto }}$} & \multicolumn{3}{|c|}{ mAP areo bike bird } & \multicolumn{3}{|c|}{ boat bottle bus } & car & \multicolumn{6}{|c|}{ cat chair cow table dog horse mbike person plant } & \multirow[t]{2}{*}{ sheep } & \multirow[t]{2}{*}{ sofa } & \multirow[t]{2}{*}{ train } & \multirow[t]{2}{*}{ tv } \\
\hline & & & & & & & & & & & & & & & & & \\
\hline SSD512 [22] & 76.8 & 82.484 .7 & 778.4 & 73.8 & 53.2 & 86.2 & 87.5 & 586.057 .8 & 83.172 .0 & 84.985 .2 & 83.9 & 79.7 & 50.3 & 77.9 & 73.9 & 82.5 & 75.3 \\
\hline OLOv2 [5] & 78.6 & $-\quad-$ & - & - & - & - & - & $-\quad-$ & $-\quad-$ & $-\quad-$ & - & - & - & - & - & - & - \\
\hline DSOD300 [16] & 77.7 & - & - & - & - & - & - & - & - & - & - & - & - & - & - & - & - \\
\hline CEBN & 80.8 & - & - & - & - & - & - & - & - & - & - & - & - & - & - & - & - \\
\hline CEBNet512 [26] & 82. & - & - & - & - & - & - & - & - & - & - & - & - & - & - & - & - \\
\hline \multicolumn{18}{|c|}{ Two-stage detector } \\
\hline Fast [3] & 70.0 & 77.078 .1 & | 69.3 & & 38.3 & $81.6^{\circ}$ & & & & & 76.6 & 69.9 & 31.8 & 70.1 & 74.8 & 80.4 & 70.4 \\
\hline Faster [4] & 73.2 & 76.579 & & 65 & 52.1 & 83.1 & 184 & ) & 81.96 & 84.884 .6 & 77.5 & 76.7 & 38.8 & 73.6 & 73.9 & 83.0 & 72.6 \\
\hline $101[13]$ & 76.4 & 79.880 .7 & 76.2 & 68.3 & 55.9 & 85.1 & 85.3 & 389.856 .7 & 87.869 .4 & 88.388 .9 & 80.9 & 78.4 & 41.7 & 78.6 & 79.8 & 85.3 & 72.0 \\
\hline & 74.6 & 78 & 176 & & & 81.9 & 8 & & & & & 74 & 6 & 75.3 & 72.1 & 82.6 & 81.4 \\
\hline & 80.5 & 79.987 .2 & 81.5 & 72.0 & 69.8 & 86.8 & 88.5 & 89.867 .0 & 88.174 .5 & 89.890 .6 & 79.9 & 81.2 & 53.7 & 81.8 & 81.5 & 85.9 & 79.9 \\
\hline $\mathrm{t}[10]$ & 82.7 & 85.787 .0 & 84.8 & 75.5 & 73.3 & 88.8 & 389.2 & 289.669 .8 & 87.576 .1 & 88.989 .0 & 87.2 & 86.2 & 59.1 & 83.6 & 83.4 & 87.6 & 80.7 \\
\hline Tet101 [21] & 77.0 & $-\quad-$ & - & - & - & - & - & $-\quad-$ & $-\quad-$ & $-\quad-$ & - & - & - & - & - & - & - \\
\hline CRFNet [22] & 72.2 & 72.682 .3 & 33.0 & 56.7 & 54.9 & 79.4 & 84.6 & 683.254 .3 & 77.566 .8 & 81.383 .4 & 78.2 & 79.5 & 42.7 & 69.6 & 71.1 & 81.8 & 71.0 \\
\hline & 78.1 & 78.783 & 178.8 & 71.3 & 64.4 & 86.1 & 88.0 & 87.864 . & 83.273 .6 & 85.786 .4 & 81.9 & 79.3 & 53.1 & 77.2 & 76.7 & 85.0 & 76.1 \\
\hline MLKP1C & 80.6 & 82.283 & 79.5 & 72.9 & 70.5 & 87 & 88 & 288.868 .3 & 86.374 .5 & 88.888 .7 & 82.0 & 81 & 56.3 & 84.2 & 83.3 & 85.3 & 79.7 \\
\hline$[20]$ & 76.8 & 74.180 & 76.0 & 65.7 & 55.4 & 83 & 58 & 387.554 .2 & 87.368 .6 & 87.485 .2 & 77.2 & 77 & 40.9 & 77.8 & 77.5 & 84.6 & 70.3 \\
\hline & 77.2 & 75.984 & 77.4 & 65. & 62.7 & 86 & 87 & 589.061 & 84.571 .3 & 86.287 .1 & 78 & 79 & 51 & 77.8 & 75.6 & 83.1 & 76.3 \\
\hline K-Faster101 & 80.5 & 80.387 .0 & 79.3 & 74.5 & 68.8 & 88.9 & 88.5 & 588.966 .9 & 86.975 .2 & 88.587 .6 & 84.7 & 80.0 & 53.7 & 83.7 & 80.3 & 85.1 & 80.8 \\
\hline
\end{tabular}

Keypoint anchors provide an extra auxiliary discrimination. As shown in Table 4, our K-Faster16 and K-Faster101 achieve mAPs of $77.2 \%$ and $80.5 \%$ on the PASCAL VOC 2007 test set, respectively. Compared with the baseline Faster R-CNN, corresponding improvements of the mAPs are respectively $4.0 \%$ and $4.1 \%$. Because K-Faster shares a similar framework with Faster R-CNN, we owe the gains to the proposed keypoint anchors. In addition, K-Faster outperforms many state-ofthe-art models, including ION [18], SSD512 [23], and YOLOv2 [5]. Our ResNet101-based results are comparable to R-FCN [9], which also uses ResNet101 as the backbone network. Although CoupleNet [10], CEBNet [26], and MLKP101 [19] show their superiority in MAP, the superiority of MLKP to KFaster is insignificant. As for CoupleNet, it uses two branches, i.e., local FCN and global FCN, to improve ResNet101-based R-FCN. Compared with R-FCN, which achieves mAP of 80.5\%, CoupleNet obtains an mAP of $82.7 \%$, as shown in Table 4 . The improvement of the mAP is $2.2 \%$, which is less than the improvement from Faster101 to K-Faster101. CEBNet embeds six layers into SSD detector, including four CEB modules. Every CEB module consists of two sub-networks. Compared with SSD512, the improvement of CEBNet512 on the mAP is $5.7 \%$. However, the network of CEBNet is much more complicated than SSD.

Fig. 4 shows some results on the PASCAL VOC 2007 test set. The implementation model is KFaster16 (77.2\% mAP). A score threshold of 0.6 is used to draw the detection bounding boxes. The blue and red colors respectively show the detections launched by Faster and keypoint anchors. Fig. 5 shows the detection ratios launched by keypoint anchors on the VOC 2007 test set using the model of $\mathrm{K}$-Faster16. The detection ratio is a proportion of the number of the objects detected by keypoint anchors to the total detections. Both Fig. 4 and Fig. 5 demonstrate that keypoint anchors are helpful for object detection. 


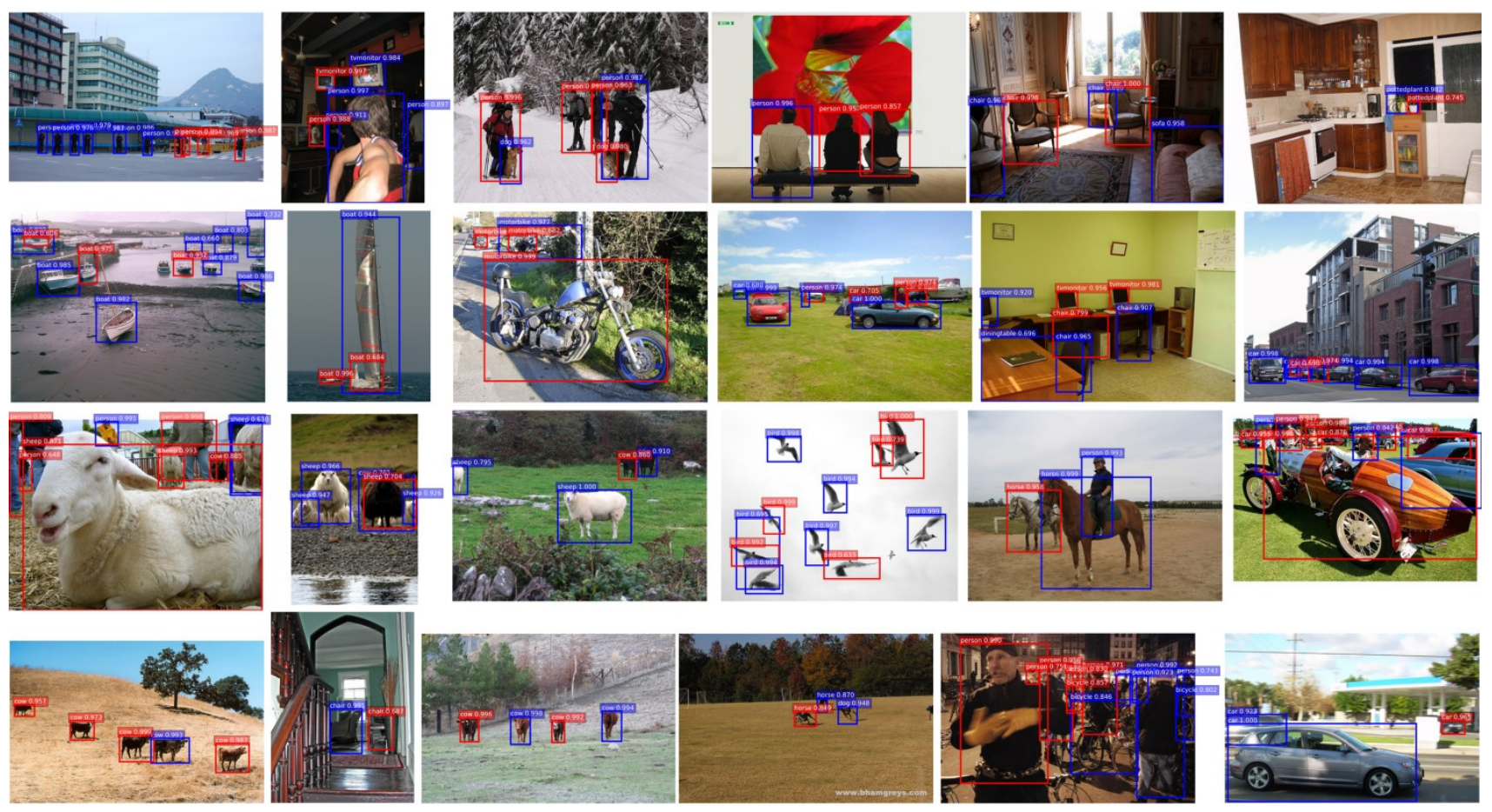

Fig. 4. Detection examples of K-Faster16 on the PASCAL VOC 2007 test set. A score threshold of 0.6 is used to draw the detection bounding boxes. The blue and red colors respectively show the detections launched by Faster and keypoint anchors.

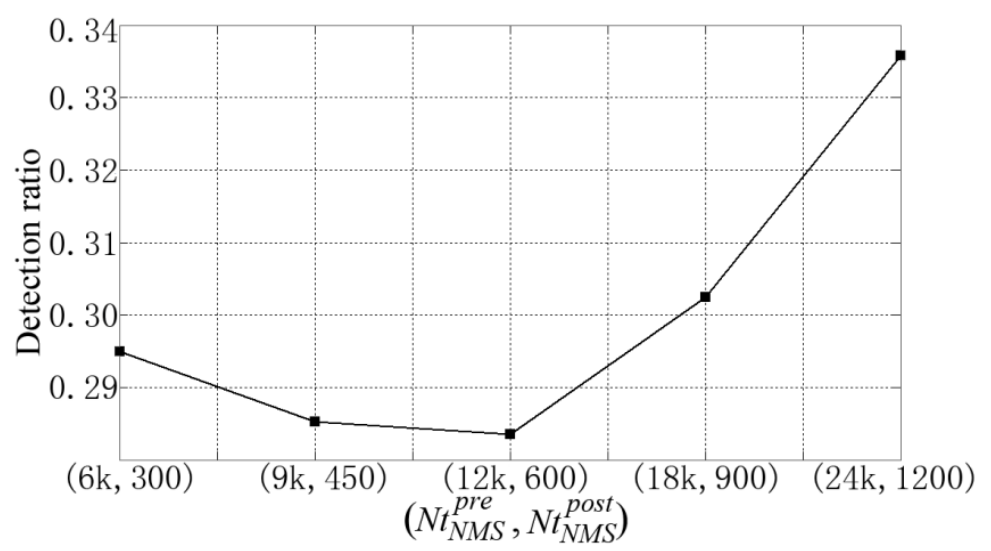

Fig. 5. Detection ratios launched by keypoint anchors on the PASCAL VOC 2007 test set.

Because the main task for object detection not only includes classification but also involves localization problem, we add an evaluation on localization. In order to investigate the positioning precision of the detection, we evaluate the mean IoUs of the true positive detections ( $\mathrm{IoU} \geq 0.5$ ). Fig. 6 shows the positioning precision on the 20 classes of the PASCAL VOC 2007 test set. Fig. 6a and Fig. $6 \mathrm{~b}$ respectively show the mean IoUs that are obtained from the backbone networks of VGG16 and ResNet101. The results of K-Faster and Faster R-CNN are shown in red and gray, respectively. The mean IoUs of K-Faster and Faster R-CNN over the 20 classes in Fig. 6a are 78.2\% and 77.8\%, respectively. The mean IoUs of K-Faster and Faster R-CNN in Fig. $6 \mathrm{~b}$ are respectively $81.7 \%$ and $81.5 \%$. Both the mean IoUs of K-Faster are greater than those of Faster R-CNN. Overall, our K-Faster improves the positioning precision of the detection. 


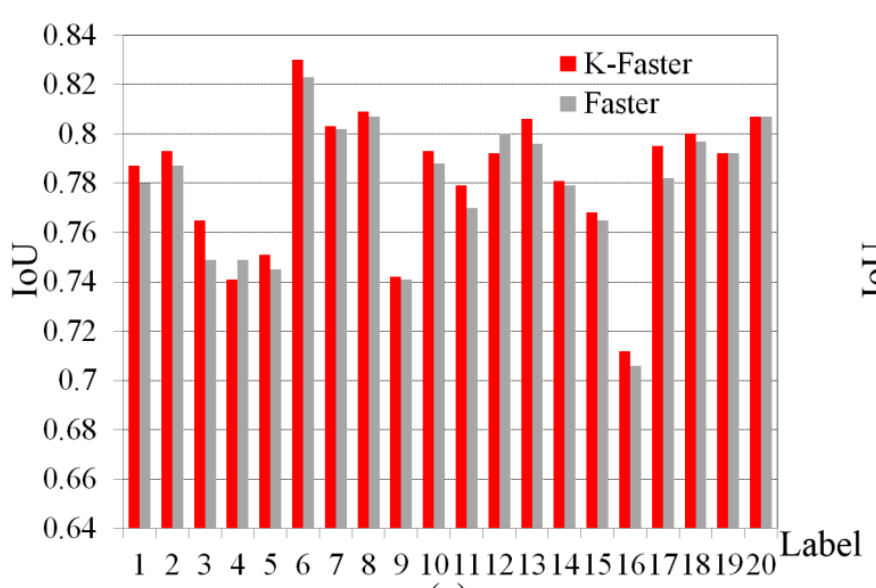

(a)

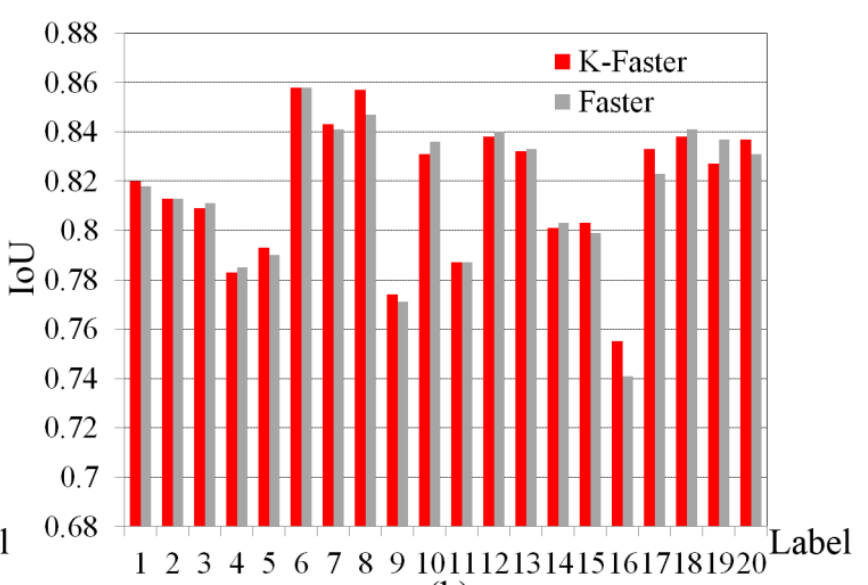

(b)

Fig. 6. The positioning precision of bounding boxes on the PASCAL VOC 2007 test set. (a) VGG16-based results. (b) ResNet101-based results.

Take Faster R-CNN as a benchmark, we evaluate the runtime of K-Faster using a dual-core i-3 4160 CPU and an NVIDIA GTX1080 GPU. We summarize the test time (ms per image) of Faster RCNN and K-Faster on the PASCAL VOC 2007 test set in Table 5. "Keypoint" and "Proposal" are respectively the runtimes in generating keypoints and proposals on CPU. "GPU" is the runtime on convolution, pooling, full-connection, and softmax layers. As shown the the total runtime in Table 5, $\mathrm{K}-\mathrm{Faster}$ is slower than Faster R-CNN for a same $N t_{N M S}^{\text {post }}$. The inferiority is largely due to the time cost on Keypoint. It takes K-Faster about $100 \mathrm{~ms}$ to generate keypoints on CPU. However, K-Faster takes advantage in GPU runtime. As shown in Table 5, the runtimes of K-Faster on GPU are 80.6, 202.0, 136.7, and $386.1 \mathrm{~ms}$. The first three GPU runtimes of K-Faster are less than those of Faster R$\mathrm{CNN}$. This is partially due to the improvement of the performance of the proposals. Overall, K-Faster is competitive against Faster R-CNN in terms of GPU runtime.

Table 5. Runtime (ms) comparisons on the PASCAL VOC 2007 test set.

\begin{tabular}{lllllll}
\hline Model & $N t_{N M S}^{\text {post }}$ & Keypoint & Proposal & GPU & Total & Rate (fps) \\
\hline Faster [4] & 300 & - & 6.2 & 90.2 & 96.4 & 10.4 \\
K-Faster16 & 300 & 107.4 & 19.7 & 80.6 & 207.7 & 4.8 \\
Faster [4] & 1200 & - & 26.2 & 216.7 & 242.9 & 4.1 \\
K-Faster16 & 1200 & 105.0 & 56.2 & 202.0 & 363.2 & 2.8 \\
Faster101 [13] & 300 & - & 6.0 & 156.1 & 162.1 & 6.2 \\
K-Faster101 & 300 & 112.5 & 18.6 & 136.7 & 267.8 & 3.7 \\
Faster101 [13] & 1200 & - & 28.8 & 383.8 & 412.6 & 2.4 \\
K-Faster101 & 1200 & 117.6 & 57.0 & 386.1 & 560.7 & 1.8 \\
\hline
\end{tabular}

As shown in Table 6, we also compare our method with the state-of-the-art methods on the test sets of VOC 2012. The K-Faster16 and K-Faster101 are run 260k and 280k training iterations, respectively. The learning rate is dropped 10 times after every $100 \mathrm{k}$ iterations on the $07++12$ dataset. Because the labels of the test set of VOC 2012 are not issued, the evaluation results in Table 6 are produced from the server of PASCAL VOC. Except for CoupleNet, K-Faster101 achieves the greatest mAP of $77.7 \%$. Compared with the standard Faster R-CNN, K-Faster16 improves the mAP of 3.5\% and K-Faster101 improves the mAP of 3.9\%. MLKP, which outperforms K-Faster on the PASCAL VOC 2007 test set, is inferior to K-Faster on the PASCAL VOC 2012 test set. Keypoint anchors are helpful for object detection on the PASCAL VOC 2012 test set. 
Table 6. Results on the PASCAL VOC 2012 test set (\%). Training data: "07++12": VOC 2007 trainval + test together with VOC 2012 trainval.

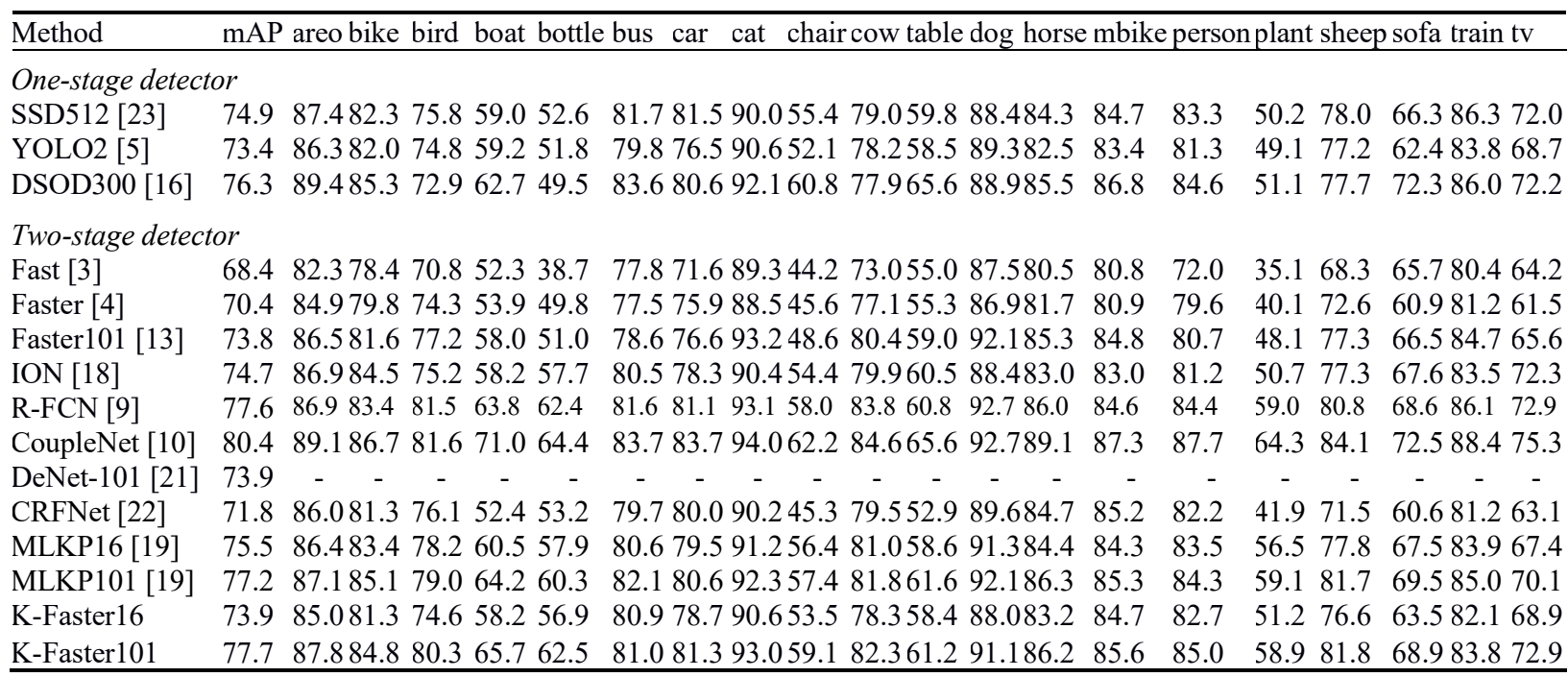

\subsection{Results on $\mathrm{MSCOCO}$}

In this Section, we present experimental results on the Microsoft COCO object detection dataset. COCO involves 80 object classes. The dataset consists of $80 \mathrm{k}$ images for training (train2014), 40k images for validation (val2014), and 20k images for testing (test-dev2015). We use the train+val (trainval) to train our model. We report COCO AP on the test-dev set, which has no public labels and requires evaluation from the server of COCO. The COCO standard metric is denoted as AP, which is the average precision evaluated at $\mathrm{IoU}$ in [0.5: 0.05: 0.95]. $\mathrm{AP}^{50}$ and $\mathrm{AP}^{75}$ are evaluated at $\mathrm{IoU}=0.50$ and 0.75 , respectively. $\mathrm{AR}^{1}, \mathrm{AR}^{10}$, and $\mathrm{AR}^{100}$ are the average recall given 1,10 , and 100 detections per image, respectively. $\mathrm{AP}^{s}, \mathrm{AP}^{m}$, and $\mathrm{AP}^{l}$ are $\mathrm{AP}$ for small $\left(\operatorname{area} \leq 32^{2}\right)$, medium $\left(32^{2}<\right.$ area $\left.\leq 96^{2}\right)$, and large (area $>96^{2}$ ) objects, respectively. $\mathrm{AR}^{s}, \mathrm{AR}^{m}$, and $\mathrm{AR}^{l}$ are the similar notations. The learning rate is initialized with 0.001 and is decayed 10 times after every 550k iterations until the iterations reach 1400k.

Table 7 shows our results on COCO. The training set trainval35k is the union of 80k train images and a random 35k subset of val images. All the results are reported on the test-dev split except for Faster-Res101*, which is reported on val split. For a fair comparison, we re-implement ResNet101based Faster R-CNN [13] on trainval split. The learning rate is initialized with 0.001 and is reduced by a factor of 10 after $600 \mathrm{k}$ iterations until the iterations reach $800 \mathrm{k}$ [37]. The test results are shown as Faster-Res $101^{\dagger}$ in Table 7. Although Table 7 lists results on three different training sets (train, trainval, trainval35k), it is reasonable to implement comparison within groups.

Table 7. Detection results on the test set of COCO (\%). *: the results are reported on val split. ${ }^{\dagger}$ : the results are reported by the re-implemented model.

\begin{tabular}{|c|c|c|c|c|c|c|c|c|c|c|c|c|c|}
\hline Method & Training set & AP & $\mathrm{AP}^{50}$ & $\mathrm{AP}^{75}$ & $\mathrm{AP}^{s}$ & $\mathrm{AP}^{m}$ & $\mathrm{AP}^{l}$ & $\mathrm{AR}^{1}$ & $\mathrm{AR}^{10}$ & $\mathrm{AR}^{100}$ & $\mathrm{AR}^{s}$ & $\mathrm{AR}^{m}$ & $\mathrm{AR}^{l}$ \\
\hline \multicolumn{14}{|l|}{ One-stage detector } \\
\hline SSD512 [23] & trainval35k & 26.8 & 46.5 & 27.8 & 9.0 & 28.9 & 41.9 & 24.8 & 37.5 & 39.8 & 14.0 & 43.5 & 59.0 \\
\hline YOLOv2 [5] & trainval35k & 21.6 & 44.0 & 19.2 & 5.0 & 22.4 & 35.5 & 20.7 & 31.6 & 33.3 & 9.8 & 36.5 & 54.4 \\
\hline DSOD300 [16] & trainval & 29.3 & 47.3 & 30.6 & 9.4 & 31.5 & 47.0 & 27.3 & 40.7 & 43.0 & 16.7 & 47.1 & 65.0 \\
\hline CEBNet300 [26] & trainval35k & 30.5 & 49.7 & - & 11.6 & 32.4 & 46.2 & - & - & - & - & - & - \\
\hline CEBNet512 [26] & trainval35k & 34.3 & 54.1 & - & 16.3 & 37.5 & 48.5 & - & - & - & - & - & - \\
\hline
\end{tabular}




\begin{tabular}{|c|c|c|c|c|c|c|c|c|c|c|c|c|c|}
\hline \multicolumn{14}{|l|}{ Two-stage detector } \\
\hline Fast [3] & train & 20.5 & 39.9 & 19.4 & 4.1 & 20.0 & 35.8 & 21.3 & 29.5 & 30.1 & 7.3 & 32.1 & 52.0 \\
\hline Faster $[4][19]$ & trainval & 21.9 & 42.7 & 23.0 & 6.7 & 25.2 & 34.6 & 22.5 & 32.7 & 33.4 & 10.0 & 38.1 & 53.4 \\
\hline Faster- Res101 [13] ${ }^{*}$ & train & 27.2 & 48.4 & - & - & - & - & - & - & - & - & - & - \\
\hline Faster-Res101 [38] & trainval35k & 30.3 & - & - & 9.9 & 32.2 & 47.4 & - & - & - & - & - & - \\
\hline Faster-Res101 [13] $]^{\dagger}$ & trainval & 31.3 & 49.6 & 33.8 & 10.3 & 35.2 & 48.2 & 28.7 & 40.8 & 41.5 & 14.2 & 47.9 & 64.6 \\
\hline ION [18] & train & 23.6 & 43.2 & 23.6 & 6.4 & 24.1 & 38.3 & 23.2 & 32.7 & 33.5 & 10.1 & 37.7 & 53.6 \\
\hline R-FCN [9] & trainval & 29.2 & 51.5 & - & 10.3 & 32.4 & 43.3 & - & - & - & - & - & - \\
\hline CoupleNet [10] & trainval & 33.1 & 53.5 & 35.4 & 11.6 & 36.3 & 50.1 & 29.3 & 43.8 & 45.2 & 18.7 & 51.4 & 67.9 \\
\hline DeNet-101 [21] & trainval & 31.9 & 50.5 & 34.2 & 9.7 & 34.9 & 50.6 & 28.4 & 39.8 & 40.3 & 13.1 & 44.8 & 64.1 \\
\hline Mask RCNN [12] & trainval35k & 38.2 & 60.3 & 41.7 & 20.1 & 41.1 & 50.2 & - & - & - & - & - & - \\
\hline CornerNet [24] & trainval35k & 40.5 & 56.5 & 43.1 & 19.4 & 42.7 & 53.9 & 35.3 & 54.3 & 59.1 & 37.4 & 61.9 & 76.9 \\
\hline CRFNet [22] & train & 23.5 & 44.3 & - & - & - & - & - & - & - & - & - & - \\
\hline MLKP16 [19] & trainval35k & 26.9 & 48.4 & 26.9 & 8.6 & 29.2 & 41.1 & 25.6 & 37.9 & 38.9 & 16.0 & 44.1 & 59.0 \\
\hline MLKP101 [19] & trainval35k & 28.6 & 52.4 & 31.6 & 10.8 & 33.4 & 45.1 & 27.0 & 40.9 & 41.4 & 15.8 & 47.8 & 62.2 \\
\hline K-Faster16 & trainval & 26.5 & 48.6 & 26.3 & 7.9 & 28.5 & 41.1 & 25.3 & 37.2 & 38.2 & 13.7 & 43.2 & 58.8 \\
\hline K-Faster101 & trainval & 34.7 & 53.8 & 37.7 & 14.1 & 37.5 & 51.0 & 30.6 & 43.9 & 44.7 & 19.7 & 49.7 & 66.0 \\
\hline
\end{tabular}

As shown in Table 7, K-Faster16 and K-Faster101 respectively achieve the APs of $26.5 \%$ and $34.7 \%$ on the trainval set. Both of them outperform corresponding standard Faster R-CNN. Corresponding APs are respectively 3.6 and 3.4 points higher than those of Faster R-CNN. Although K-Faster101 is inferior to CEBNet512, CoupleNet, and MLKP on the test set of VOC 2007 and VOC 2012 (Tables 4 and 6), K-Faster101 outperforms them on the test set of COCO (Table 7). It should be noted that K-Faster cannot outperform Mask R-CNN and CornerNet on COCO. Mask R-CNN is a kind of segmentation method that is designed for pixel-to-pixel alignment using FPN-based ResNet101. The advantage of Mask R-CNN on box detection is partially due to the benefits of segmentation branch and multi-task training [12]. Because CornerNet integrates many technologies in training, it takes advantage in high performance. Besides multi-stream technology is employed to design CornerNet, data augmentation techniques and Principal Component Analysis (PCA) are applied to the input images. In addition, CornerNet uses an optimized training loss for training. It seems that a method integrating several technologies is in favor of a high performance. Although KFaster cannot outperform all the listed state-of-the-art methods, Keypoint anchors are helpful for object detection for the more challenging COCO dataset.

\section{Conclusions}

In this paper, a local keypoint-based Faster R-CNN is proposed. The 2-combinations of the produced keypoints are selected to generate anchors. An area-based technology is designed to encode the keypoint anchors with a heterogeneous distribution. The keypoint anchors are coupled with Faster anchors to improve object detection. With the coupling anchors, our K-Faster approach not only increases the mAP performance but also improves the positioning precision of the detected boxes. In the future work, we first plan to improve detection performance using geometry knowledge since knowledge, such as global structure and context, may be helpful for detection. Second, we plan to improve CNN-based method with the help of intuitionistic fuzzy set. We hope to annotate object using membership and non-membership classifications and design dual-network for object detection.

\section{Acknowledgment}

This work was supported by the Anhui Provincial Natural Science Foundation (1808085MF171, 1708085MF145), the National Natural Science Foundation of China (61672039, 61573023, 61602009), and the University of Hull 'Evolving a Plastics Circular Economy' project funded by the EPSRC (EP/S025 537/1).

\section{References}


[1] Cai Z, Vasconcelos N (2018) Cascade R-CNN: Delving into high quality object detection. In: Proceedings of the IEEE conference on computer vision and pattern recognition, pp 6154-6162

[2] Krizhevsky A, Sutskever I, Hinton GE (2017) ImageNet classification with deep convolutional neural networks. Commun ACM 60(6):84-90

[3] Girshick R (2015) Fast R-CNN. In: Proceedings of the IEEE international conference on computer vision, pp 14401448

[4] Ren S, He K, Girshick R, Sun J (2017) Faster R-CNN: Towards real-time object detection with region proposal networks. IEEE Trans Pattern Anal Mach Intell 39(6):1137-1149

[5] Redmon J, Farhadi A (2017) Yolo9000: better, faster, stronger. In: Proceedings of the IEEE conference on computer vision and pattern recognition, pp 7263-7271

[6] Lin TY, Maire M, Belongie S, Hays J, Perona P, Ramanan D, Dollár P, Zitnick CL (2014) Microsoft COCO: Common objects in context. In: Proceedings of the European conference on computer vision, pp 740-755

[7] Russakovsky O, Deng J, Su H, Krause J, Satheesh S, Ma S, Huang Z, Karpathy A, Khosla A, Bernstein M, et al. (2015) Imagenet large scale visual recognition challenge. Int J Comput Vis 115(3):211-252

[8] Everingham M, Eslami SA, Van Gool L, Williams CK, Winn J, Zisserman A (2015) The pascal visual object classes challenge: A retrospective. Int J Comput Vis 111(1):98-136

[9] Dai J, Li Y, He K, Sun J (2016) R-FCN: Object detection via region-based fully convolutional networks. In: Advances in neural information processing systems, pp 379-387

[10] Zhu Y, Zhao C, Wang J, Zhao X, Wu Y, Lu H (2017) CoupleNet: Coupling global structure with local parts for object detection. In: Proceedings of the IEEE international conference on computer vision, pp 4126-4134

[11] Simonyan K, Zisserman A (2015) Very deep convolutional networks for large-scale image recognition. In: International conference on learning representations

[12] He K, Gkioxari G, Dollár P, Girshick R (2017) Mask R-CNN. In: Proceedings of the IEEE international conference on computer vision, pp 2961-2969

[13] He K, Zhang X, Ren S, Sun J (2016) Deep residual learning for image recognition. In: Proceedings of the IEEE conference on computer vision and pattern recognition, pp 770-778

[14] Bai Y, Zhang Y, Ding M, Ghanem B (2018) SOD-MTGAN: Small object detection via multi-task generative adversarial network. In: Proceedings of the European conference on computer vision, pp 206-221

[15] Liu L, Ouyang W, Wang X, Fieguth P, Chen J, Liu X, Pietikäinen M (2018) Deep learning for generic object detection: A survey. arXiv preprint arXiv: 180902165

[16] Shen Z, Liu Z, Li J, Jiang YG, Chen Y, Xue X (2017) DSOD: Learning deeply supervised object detectors from scratch. In: Proceedings of the IEEE international conference on computer vision, pp 1919-1927

[17] Girshick R, Donahue J, Darrell T, Malik J (2014) Rich feature hierarchies for accurate object detection and semantic segmentation. In: Proceedings of the IEEE conference on computer vision and pattern recognition, pp 580-587

[18] Bell S, Lawrence Zitnick C, Bala K, Girshick R (2016) Inside-outside net: Detecting objects in context with skip pooling and recurrent neural networks. In: Proceedings of the IEEE conference on computer vision and pattern recognition, pp 2874-2883

[19] Wang H, Wang Q, Gao M, Li P, Zuo W (2018) Multi-scale location-aware kernel representation for object detection. In: Proceedings of the IEEE conference on computer vision and pattern recognition, pp 1248-1257

[20] Li H, Liu Y, Ouyang W, Wang X (2019) Zoom out-and-in network with map attention decision for region proposal and object detection. Int J Comput Vis 127(3):225-238

[21] Tychsen-Smith L, Petersson L (2017) DeNet: Scalable real-time object detection with directed sparse sampling. In: Proceedings of the IEEE international conference on computer vision, pp 428-436 
[22] Jiang L, Zhong W, Ji J, Xiong H (2019) Object codetection based on a higher-order conditional random field. J Electron Imaging 28(2):023019

[23] Liu W, Anguelov D, Erhan D, Szegedy C, Reed S, Fu CY, Berg AC (2016) SSD: Single shot multibox detector. In: Proceedings of the European conference on computer vision, pp 21-37

[24] Law H, Deng J (2018) CornerNet: Detecting objects as paired keypoints. In: Proceedings of the European conference on computer vision, pp 734-750

[25] Lin TY, Goyal P, Girshick R, He K, Dollár P (2017) Focal loss for dense object detection. In: Proceedings of the IEEE international conference on computer vision, pp 2980-2988

[26] Chen Y, Zhao M, Tan X, Tang H, Sun D (2019) Accurate and efficient object detection with context enhancement block. In: 2019 IEEE international conference on multimedia and expo (ICME), pp 1726-1731

[27] Wei XS, Luo JH, Wu J, Zhou ZH (2017) Selective convolutional descriptor aggregation for fine-grained image retrieval. IEEE Trans Image Process 26(6):2868-2881

[28] Tran CK, Tseng CD, Chao PJ, Ting HM, Chang L, Huang YJ, Lee TF (2017) Local intensity area descriptor for facial recognition in ideal and noise conditions. J Electron Imaging 26(2):023011

[29] Lowe DG (2004) Distinctive image features from scale-invariant keypoints. Int J Comput Vis 60(2):91-110

[30] Bay H, Ess A, Tuytelaars T, Van Gool L (2008) Speeded-up robust features (SURF). Comput Vis Image Underst 110(3):346-359

[31] Liu H, Li L, Wang Z, Huo Z (2017) STBD: A simple tri-bit binary descriptor for point matching. IET Comput Vis 11(8):691-701

[32] Lei Z, Pietikäinen M, Li SZ (2013) Learning discriminant face descriptor. IEEE Trans Pattern Anal Mach Intell 36(2):289-302

[33] Duan Y, Lu J, Feng J, Zhou J (2017) Learning rotation-invariant local binary descriptor. IEEE Trans Image Process 26(8):3636-3651

[34] Ding X, Luo Y, Yi Y, Jie B, Wang T, Bian W (2016) Orthogonal design for scale invariant feature transform optimization. J Electron Imaging 25(5):053030

[35] Jia Y, Shelhamer E, Donahue J, Karayev S, Long J, Girshick R, Guadarrama S, Darrell T (2014) Caffe: Convolutional architecture for fast feature embedding. In: Proceedings of the 22nd ACM international conference on multimedia, pp 675-678

[36] Abadi M, Barham P, Chen J, Chen Z, Davis A, Dean J, Devin M, Ghemawat S, Irving G, Isard M, et al. (2016) TensorFlow: A system for large-scale machine learning. In: 12th USENIX symposium on operating systems design and implementation, pp 265-283

[37] Chen X, Gupta A (2017) An implementation of Faster R-CNN with study for region sampling. arXiv preprint arXiv: 170202138

[38] Li Z, Peng C, Yu G, Zhang X, Deng Y, Sun J (2017) Light-head R-CNN: In defense of two-stage object detector. arXiv preprint arXiv: 171107264 